\title{
A study of the unification of quiet-Sun transient-event phenomena
}

\author{
R. A. Harrison ${ }^{1}$, L. K. Harra ${ }^{2}$, A. Brković ${ }^{3}$, and C. E. Parnell ${ }^{4}$ \\ 1 Space Science and Technology Dept., Rutherford Appleton Laboratory, Chilton, Didcot, \\ Oxfordshire OX11 0QX, England, and Honorary Visiting Professor, School of Mathematics and Statistics, \\ the University of St Andrews, UK \\ 2 Mullard Space Science Laboratory, University College London, Holmbury St Mary, Dorking Surrey RH5 6NT, UK \\ 3 Kiepenheuer-Institut für Sonnenphysik, 79104-Freiburg, Germany \\ ${ }^{4}$ School of Mathematics and Statistics, The University of St Andrews, St Andrews, Fife KY16 9SS, UK
}

Received 2 December 2002 / Accepted 9 July 2003

\begin{abstract}
A number of small-scale, globally distributed solar transient event-types have been reported in the literature. Their potential role in fundamental processes in the solar atmosphere, such as coronal heating and wind acceleration, is under active investigation. However, the event-types, such as those known as blinkers, explosive events, EUV (extreme-UV) network and cell brightenings, network flares, heating events, nanoflares and EUV brightenings are basically classifications which are driven to a large extent by different observational techniques and different instruments rather than the identification of a clear differing physical phenomenon. We investigate the different instrumental and technique limitations and attempt to identify any unification of the reported quiet-Sun transient, small-scale phenomena. We find that once observational techniques have been considered, a number of the different classifications appear to be the same. This suggests that events known as blinkers, network and cell brightenings and EUV brightenings are the same event-type. We suggest that the term blinker be used as a generic term to describe these events. However, there appears to be little evidence that blinkers and explosive events are directly related. Furthermore, although a small percentage of blinkers and nanoflares/heating events appear to be related to one another, these events pose a number of important questions suggesting that either (i) blinkers and nanoflare/heating events are all created by the same mechanism, i.e. for some blinker events, the conditions are such that higher temperatures are found, or (ii) there are two types of event, including the "traditional" blinker which is effectively a transition region brightening driven by a density or filling factor enhancement, and a mini-flare-like event which reaches higher temperatures, presumably driven by reconnection.
\end{abstract}

Key words. Sun: activity - Sun: atmosphere - Sun: UV radiation

\section{Introduction}

There is a great need to identify fundamental phenomena which occur in the solar atmosphere, and to identify their roles in important processes such as coronal heating, wind acceleration, mass ejection and flare activity. It has been recognised that small-scale quiet-Sun transient events, which occur over the entire solar disc, may well provide direct evidence for processes such as magnetic reconnection, plasma acceleration and heating. In particular, the many reports of differing quietSun event-types has produced a complex view of the solar atmosphere. This is because the classification of these many event-types is driven perhaps more by instrumental limitations and observational techniques than by differing physical processes. These event-types include explosive events (e.g. Innes 2001), blinkers (e.g. Harrison et al. 1999), cell and network brightenings (e.g. Harra et al. 2000), network flares, heating

Send offprint requests to: R. A. Harrison,

e-mail: r.harrison@rl.ac.uk events and nanoflares (e.g. Krucker \& Benz 1998; Benz \& Krucker 1999; Parnell \& Jupp 2000; Aschwanden \& Parnell 2002), and even within studies apparently of the same eventtypes, different instruments or observing techniques serve to complicate the picture. Indeed, are all the event-types listed above simply the same phenomenon viewed in different ways? If not, are we able to distinguish between fundamentally different event-types and perhaps unify some of the different classes of event?

It is the purpose of this paper to review the quiet-Sun transient-event "zoo" with a view to confirming or denying the unification of particular event-types. It is not the purpose of this paper to present a unified model, but to demonstrate that operational and instrumental differences have contributed significantly to the classification of a number of small-scale solar phenomena which are most probably identical. Having said that, some comments on the links between proposed models will be made during this study. 
The basic desire, then, is one of clarification, to pave the way for a better understanding of quiet-Sun fundamental processes and, in particular, to aid the modelling of these processes.

\section{EUV brightenings at transition region temperatures}

There are many observations of brightenings detected at transition region temperatures, using a variety of observational techniques, and analysed with a variety of different methods. Thus, we have a complex picture of transient brightenings with differing results and interpretations. We now examine these different observations to establish the links between them.

The first event-type we consider is the blinker, which is characterised as a small-area brightening in the extremeultraviolet (EUV). Such events have been studied using different EUV instruments aboard the Solar and Heliospheric Observatory (SOHO), though past observations from Skylab were most likely the first to view this solar phenomenon (see Sect. 1 of Harrison et al. 1999).

Harrison et al. (1999) studied the characteristics 97 blinkers in the quiet Sun, in a comprehensive follow-up study to the original announcement of EUV blinkers using observations from the Coronal Diagnostic Spectrometer aboard SOHO by Harrison (1997). The blinkers are basically identified as flashes in EUV emission lines, especially in the transition region lines of O III, IV and V, which have characteristic temperatures of $100000 \mathrm{~K}, 160000 \mathrm{~K}$ and $250000 \mathrm{~K}$, respectively. Few events showed high temperature signatures ( 1 million $\mathrm{K}$ plus) and the signatures at cooler temperatures (specifically $20000 \mathrm{~K}$ ) were less evident.

The most basic results of the Harrison et al. (1999) study indicate that typically a blinker shows intensity enhancements of up to $60 \%$, with durations commonly in the range 400-1600 s. The analysis suggested a global blinker birth rate of $1.24 \mathrm{~s}^{-1}$, with some 3000 in progress at any point in time on the solar surface. The blinker events analysed by Harrison et al. (1999) and by Harrison (1997) were identified by sight, i.e., by visual scanning of the data to identify pixel groups with enhancements in intensity, for further detailed analysis.

Although no specific analysis of the location of each blinker with respect to the network was performed as part of this work, the visual identification method resulted in the authors claiming that the events were predominantly found in the network.

Emission line ratio analyses of the blinkers showed that they are due to either density or filling factor enhancements rather than to changes in temperature. This was explained through the merging of field lines in the network regions.

The work has been extended considerably by Bewsher et al. (2002) whose most significant advance was the routine, automated identification of blinkers. This revealed far more events than the previous studies, allowing a superior statistical analysis, and confirmed and extended the findings. In their detection routine, Bewsher et al. (2002) looked for pixel groups that contained a minimum of $n_{\mathrm{p}}$ pixels which simultaneously peaked with enhancements of at least $n_{\lambda} \lambda$ above the background; $\lambda$ was defined for each wavelength as the value
Table 1. The properties of EUV blinkers due to Bewsher et al. (2002)

\begin{tabular}{ll}
\hline \hline Property & Value \\
\hline Global frequency & $10-20 \mathrm{~s}^{-1}$ \\
Mean intensity enhancement $(\mathrm{O} \mathrm{V})$ & $70-80 \%$ \\
Mean Area & $2-3 \times 10^{7} \mathrm{~km}^{2}$ \\
Mean Lifetime & $16.4 \mathrm{~min}$ \\
\hline
\end{tabular}

below which $99 \%$ of the errors exist. Furthermore, $n_{\lambda}$ was chosen to be anything between 3 and 10. i.e., peaks between $9 \sigma$ and $30 \sigma$ were sought! Hence, the peaks that they identified are considerably larger than those typically taken when looking, for instance, at nanoflares. In the $\mathrm{O} \mathrm{V}$ line, which is a strong transition region line, many blinkers were found. However, in the weaker chromospheric and coronal lines not surprisingly fewer events of such size were found. In He I, which is at a temperature of $20000 \mathrm{~K}$, approximately $25 \%$ of the O V blinkers had strong He I signatures with peaks of size $5 \lambda_{\mathrm{HeI}}, 50 \%$ had peaks of size $3 \lambda_{\mathrm{He} \mathrm{I}}$ and $90 \%$ had peaks of just $\lambda_{\mathrm{HeI}}$. In the coronal lines, even fewer significant peaks were found coincident with the $\mathrm{O} \mathrm{V}$ blinkers with just $8 \%$ of the $\mathrm{Mg}$ IX and $13 \%$ of the $\mathrm{Mg} \mathrm{X}$ having peaks greater than the $\lambda$ for each line. Despite the greater intensity of the $\mathrm{O} V$ events detected the basic parameters agreed well with those for the blinkers seen by Harrison et al. (1999). Thus, we now consider the basic parameters which best describe EUV blinkers as those of Table 1, where the global frequency of $20 \mathrm{~s}^{-1}$ refers to blinkers with peaks of at least $3 \lambda$ and a minimum size of $3 \mathrm{CDS}$ pixels $\left(3.5 \times 10^{6} \mathrm{~km}^{2}\right)$.

A second paper by Parnell et al. (2002) used an identical approach to the above to study blinker activity in active regions. The results were very similar, except for a slightly larger enhancement and global frequency. Thus, there appears to be no fundamental difference between the blinker activity of the quiet Sun and active regions.

Bewsher et al. (2002) addressed the location question specifically. Although they showed that the blinkers are strongly associated with the network, they showed also that they can occur at any location (network, cell centres etc). Indeed, Parnell et al. (2002) detected some events that occurred over the penumbra and umbra of sunspots, as well as plage regions.

All of these studies (Harrison 1997; Harrison et al. 1999; Bewsher et al. 2002; Parnell et al. 2002) made use of small area rastered images using the Coronal Diagnostic Spectrometer (CDS; Harrison et al. 1995). CDS is a spectrometer, which produces a spectral image for each exposure using, in this case, a $4 \operatorname{arcsec} \times 240$ arcsec slit. The spectrometer is stigmatic, providing 1.68 arcsec image pixels along the slit. Scanning across an area of the Sun, using an internal mirror, allows a series of exposures to build up an image in each of the selected emission lines. The technique provides the spectral line intensities and shapes, for the plasma diagnostic analyses, but the scanning takes time. Each rastered image returned to Earth for these studies was of size $40 \operatorname{arcsec} \times 100 \operatorname{arcsec}$ or 
$40 \operatorname{arcsec} \times 120$ arcsec (see Table 1 in Harrison et al. 1999) and, with the interlacing of $10 \mathrm{~s}$ or $15 \mathrm{~s}$ exposures with the mirror movements, the image cadences were $151 \mathrm{~s}$ and $194 \mathrm{~s}$. This places a lower limit on the duration of identifiable blinkers at roughly twice this value.

Berghmans et al. (1998) also produced a study of EUV brightenings, and in their case the data were a series of images with no spectral capability. They used the Extreme Ultraviolet Imaging Telescope (EIT; Delaboudiniere et al. 1995) to produce few-hour, part-Sun EUV image sequences using filters providing two bands centred on the He II $304 \AA$ line $(80000 \mathrm{~K})$ and the Fe XII $195 \AA$ line $(1600000 \mathrm{~K})$. The two filter sequences were run at different times, directed at Sun centre with an area of $600000 \mathrm{~km} \times 600000 \mathrm{~km}$, with cadences of about $1 \mathrm{~min}$, and duration 3 hours $10 \mathrm{~min}$ for the He II and 1 hour 5 min for the Fe XII. The sequences revealed many brightening events. Berghmans et al. (1998) suggested that the He II brightenings were the EIT signature of the blinkers reported by Harrison (1997). Although the He II brightenings were from plasma much cooler than the most significant blinkers discussed above, there are a number of reasons to believe that they are one and the same.

Berghmans et al. (1998) calculated a global birth rate for the He II events. As for the blinkers observed by Bewsher et al. (2002), their figure was dependent on the definition of an acceptable brightening, i.e. its intensity departure from background. Increases to 2.5-3 sigma above background produced global rates of $15-20 \mathrm{~s}^{-1}$. Bewsher et al. (2002) used a different approach (summarised in their Table I) which gives values in the range $10-20 \mathrm{~s}^{-1}$ for events observed in $\mathrm{O} \mathrm{V}$ with increases between 9-15 sigma. Using the fact that $90 \%$ of $\mathrm{O} \mathrm{V}$ blinkers have weak ( 3 sigma) enhancements in He I the results from Berghmans et al.'s study are consistent with those from Bewsher et al. (2002). This is quite remarkable, especially when one considers that they are from fundamentally different instruments with different sensitivities, different observational procedures and different wavelengths (temperatures).

The He II blinkers were found to have durations from the (observational) lower limit of $120 \mathrm{~s}$ to several hours. Berghmans et al. (1998) found that the durations could be plotted as a power law of index approximately 3.1, as a function of global occurrence rate. The distribution flattens off at durations under about $10 \mathrm{~min}$. Although their analysis is not directly comparable to the Bewsher et al. (2002) work, it is clear that the vast majority of the He II blinkers have durations in the range 2-20 min. Noting the lower duration cut off of the CDS blinker observations (5 min), the durations of the EIT He II and the CDS blinkers are effectively identical.

Berghmans et al. (1998) also plotted distributions of areas and peak intensities for their events. The majority of their events had areas in the range $1-10 \times 10^{7} \mathrm{~km}^{2}$, which, again, is consistent with the results of Bewsher et al. (2002) for the blinker events. The peak intensities of the He II events were plotted as a distribution of intensities above background. This is difficult to compare to the CDS blinker figures, which were given as percentage increases from background, without knowledge of the EIT background levels. However, if the background level of Berghmans et al.'s Fig. 12 is typical, we are seeing very similar increases in intensity, with most events in the range $30-100 \%$ increases in intensity.

Finally, with regard to the EIT He II events, it was shown that the brightenings were found not only in the network, but also in cell interiors. This was contrary to the original announcement of blinkers (Harrison 1997) but consistent with the subsequent, more extensive CDS blinker studies (e.g. Bewsher et al. 2002).

We may, therefore, conclude that the EIT He II brightenings and blinkers are most likely the same event type.

Similar events have been discussed under the titles "quietSun cell and network brightenings" by Harra, Gallagher and colleagues (Harra et al. 2000; Gallagher et al. 1999). These are EUV brightenings, again, detected using the CDS instrument and the results suggest that many small-scale events occur inside network cells and not just at the edges where the initial CDS observations of blinkers were found. Harra and co-workers decided to classify the events as network or cell brightenings, depending on location, partly because the original blinker announcement suggested that blinkers were a network phenomena, but also because they felt that the processes in the network and cell regions may be different. However, we note that the subsequent, more detailed and more extensive blinker studies due to Bewsher et al. (2002) and Parnell et al. (2002) have shown that the original blinker events are indeed found in the cell and network regions, though the latter is more common.

In all, Harra et al. (2000) found 1125 brightening events. Their observation scheme was very different to those discussed in the CDS studies above. They used the CDS $4 \operatorname{arcsec} \times$ 240 arcsec slit, pointed to a particular quiet-Sun location, and, without rastering, simply took $15 \mathrm{~s}$ exposures for 1 hour $53 \mathrm{~min}$ on the same location. This "single slit location" scheme does not build up an image, but it allows a cadence, which is near to the exposure time, i.e. an order of magnitude better than the Harrison et al. (1999) studies. The stigmatic nature of the CDS instrument means that there is spatial information, but in one direction only, with pixels of 1.68 arcsec. Data were returned across two emission lines, namely the $250000 \mathrm{~K} \mathrm{O} \mathrm{V}$ line at $629 \AA$ and the $20000 \mathrm{~K} \mathrm{He}$ I line at $584 \AA$. Both of these lines were used in the previous studies, though Harra et al. (2000) only analysed the O V data. The slit was directed to one location (oriented in the $\mathrm{N}-\mathrm{S}$ direction), without solar feature tracking, that is, the Sun rotated through the slit image. The slit was directed towards Sun centre and the solar rotation rate at that location was of order 9 arcsec/hour.

As mentioned, brightenings were detected in the cell and network regions. The basic, mean parameters of the cell and network events are reproduced (from Harra et al. 2000, Table 1), in Table 2.

This table must be compared to Table 1, to compare these brightenings to the blinkers detected using rastered observations by Harrison, Bewsher, Parnell and co-workers. The most straightforward parameter to compare is the duration. Harra et al.'s events all lay in the duration range $50 \mathrm{~s}$ to $500 \mathrm{~s}$, with the majority of events in the $70 \mathrm{~s}$ to $300 \mathrm{~s}$ range. These times are a factor of 2 or more shorter than the average event duration found by Bewsher et al. (2002) of $16.4 \mathrm{~min}$. If the events are 
Table 2. The properties of the cell and network brightenings due to Harra et al. (2000).

\begin{tabular}{llll}
\hline \hline Property & Network & Cell & Total \\
\hline Global frequency & $2177 \mathrm{~s}^{-1}$ & $2635 \mathrm{~s}^{-1}$ & $2292 \mathrm{~s}^{-1}$ \\
Mean O V enhancement & $10 \%$ & $11 \%$ & $11 \%$ \\
Mean lifetime & $150 \mathrm{~s}$ & $96 \mathrm{~s}$ & $100 \mathrm{~s}$ \\
\hline
\end{tabular}

the same, simply viewed using a different procedure, then we must reconcile these differences.

The first point to note is the fact that solar rotation will influence the durations found by Harra et al. (2000). This is a fact of which Harra et al. (2000) were well aware. The rotation will have truncated some events, with the longer duration events suffering the most. For example, if we consider an event of size one pixel, for a 3 min duration starting when the brightening area is covered exactly by the slit width, some $10 \%$ of the brightening area is lost to the image by the end of the event. For similar events of duration, 6,15 and $21 \mathrm{~min}$, the same analysis provides figures of $20 \%, 50 \%$ and $70 \%$. Thus, any event of duration above several minutes will suffer truncation, and may be counted as a shorter duration event in the analysis. Of course, the rotation dictates that event durations longer than a particular value cannot be recorded; Harra et al.'s maximum duration was $500 \mathrm{~s}$.

On the other hand, the rastering technique of the earlier studies resulted in a minimum duration recorded of $300 \mathrm{~s}$. There is no doubt that the technique employed by Harra et al. (2000) would reveal many shorter duration events, and thus bring the average event duration down significantly.

Are these arguments sufficient to reconcile the $100 \mathrm{~s}$ mean duration with the previous 16.4 min duration? This is impossible to confirm, but one would certainly expect the single-slit observations to have a much shorter duration average than the rastered observations if the two observation schemes are looking at the same solar event-types. In a sense, the blinkers identified using the rastering techniques were well suited to studies of event sizes and locations, and the single slit studies best suited to temporal studies, but the outcome of these two techniques was to produce two data-sets, in terms of event duration, with very little temporal overlap!

The Harra et al. (2000) study, then, concentrated on the shorter duration events, and found many. The global frequencies are much greater than those calculated in the previous studies, which is not surprising due to the lower threshold value and the capability for identification of shorter duration events.

The single slit and raster observations of these events, using CDS, are exclusive to the extent that it is not really possible, using these data alone to confirm or deny that they are detecting the same event-types. For this, we have to refer to a third technique, through the work of Brković et al. (2001). However, the single slit work does confirm that there are brightenings in the EUV region both in network and cell regions, and this is in keeping with the previous studies.

The CDS observations of EUV brightenings discussed above make use of small rastered images or single slit
Table 3. The properties of EUV brightenings from open slit observations by Brković et al. (2001).

\begin{tabular}{ll}
\hline \hline Property & Value \\
\hline Global frequency & $22.1 \mathrm{~s}^{-1}$ \\
Mean intensity enhancement (O V) & $65 \%$ \\
Mean area & $2.2 \times 10^{7} \mathrm{~km}^{2}$ \\
Mean lifetime & $15.05 \mathrm{~min}$ \\
\hline
\end{tabular}

observations. Brković et al. (2001) made use of another approach. They used the CDS "open" slit, which allows instantaneous snapshot images of a $90 \operatorname{arcsec} \times 240$ arcsec region of the Sun for well-separated, bright emission lines. They made use of the He I $584 \AA$, O V $629 \AA$ and Mg IX $368 \AA$ lines. This wide-slit technique removes the spectral capability of CDS, but allows rapid sequences to be made. In this case, the exposure time was $25 \mathrm{~s}$, which, with the read-out overhead, resulted in a cadence of $31 \mathrm{~s}$ for two periods of about 4 hours each, in December 1996. A solar feature tracking operation was used and, when corrections were made to ensure that each pixel viewed the same part of the Sun for the entire 4 hour sequence in each case, the usable area was $67 \operatorname{arcsec} \times 217$ arcsec, with $1.68 \operatorname{arcsec} \times 1.68$ arcsec pixels.

Brightenings were identified and the $\mathrm{O} V$ characteristics are summarised in Table 3.

These parameters agree very well with those given in Table 1 even though both the observational technique and the detection algorithm for events is quite different to Bewsher et al. (2002), Harrison (1997) and Harrison et al. (1999). One must conclude from this, as did Brković et al. (2001), that the events being described were those described as blinkers in the earlier studies.

However, it is noted that the cadence of $31 \mathrm{~s}$ allows, in principle, the detection of event durations of down to $62 \mathrm{~s}$, and this is well within the duration range of the Harra et al. (2000) single slit events. Indeed, Brković et al. (2001) note that although they should in principle detect events of order down to $62 \mathrm{~s}$, if they exist, their events were in the range $3 \mathrm{~min}$ to $110 \mathrm{~min}$. Their event distribution peaks at $10 \mathrm{~min}$, with the average at $15.05 \mathrm{~min}$. The detection routine used by Brković et al. (2001) tends to count several multiple small peaks as one large event and is therefore possibly biased towards longer duration events. It may also be that there really are fewer very short lived events. However, it is the short duration range that is explored using the single-slit approach of Harra et al. (2000).

To confirm that the events detected by Harra et al. (2000) and Brković et al. (2001) are indeed the same, the automated technique that was used in Harra et al. (2000) was carried out on Brković et al's dataset. To mimic the single-slit approach one pixel column was extracted from the $90 \times 240$ arcsec frames (Col. 25 in the solar E-W direction) and the data analysed. It is important to note that this "single slit" is not fixed, but feature tracks. Hence, if the feature tracking really keeps up with the rotation of the Sun, we should actually see event lifetimes which are more realistic than those of the original Harra et al. (2000) study. 

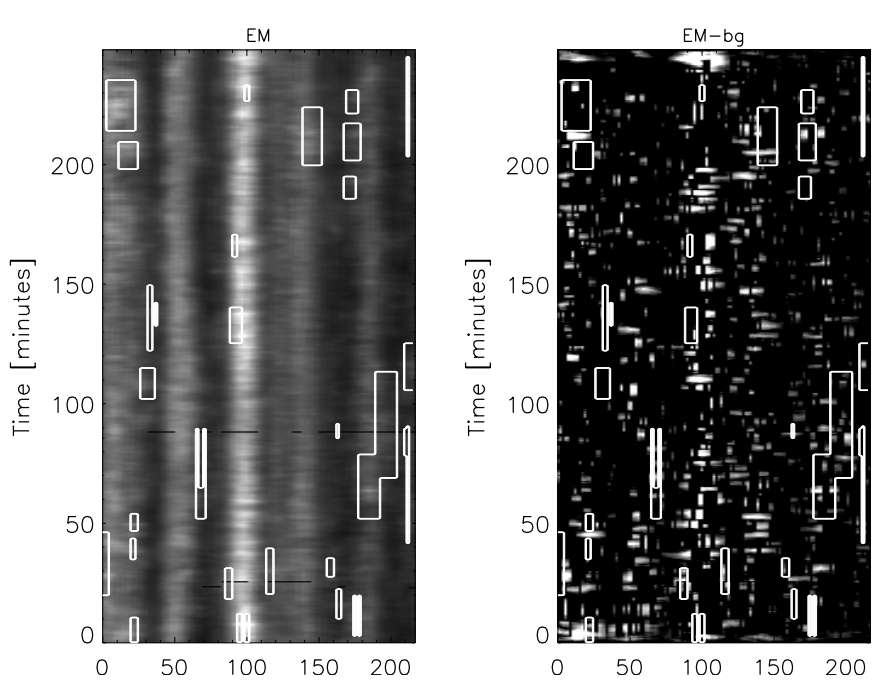

Fig. 1. An analysis of the Brković et al. (2001) wide-slit data-set using the single-slit analysis technique due to Harra et al. (2000). The lefthand panel shows the emission measure variation over time ( $y$-axis), and along the slit ( $x$-axis). The brighter regions clearly denote the network. The right-hand panel shows the same figure but following the background removal. Many small-scale brightenings can be seen in both the network and cell regions. The boxes denote the timing and location of events detected using Brković's study.

The left-hand panel of Fig. 1 shows the raw pseudo singleslit data, displayed with time along the $y$-axis and slit length along the $x$-axis. The image is dominated by the network structure, which shows smooth variation with time. The smoothvarying background was removed by examining the intensity curves of pixels. Intensity minima for pixels were determined by finding times when intensities were less than three adjacent intensities, on either side. The result of this background removal on the Brković et al. data is shown in the right-hand panel of Fig. 1. Thus, the bright features of the right-hand image are the transient brightenings that would have been identified by using the Harra et al. technique, but using the Brković et al. (2001) data-set.

From the results of Brković et al. (2001) we would expect about 26 events to be detected in this single slit approach. However, clearly the Harra et al. technique has detected far more events. This is to some extent not surprising since the analysis techniques used are very different. The Harra et al. technique counts events with intensity enhancements of just $10 \%$ whereas the Brković et al. technique only counts events with enhancements reaching over $45 \%$.

In Fig. 1, the boxed areas show the location and timing of the events detected by Brković et al. for the same data-set. This amounts to 30 events, which is consistent with what we expect. The brightenings identified by the Harra et al. background subtraction of the right hand panel of Fig. 1 are greater in number and many are clearly of short duration. Thus, the figure illustrates well the output of the different techniques.

In addition, the figure does show some events detected by Brkovoć et al. with no apparent Harra et al. event. The Brković et al. approach uses a running monitor of the intensities looking for enhancements over a "local" background level at a $45 \%$ threshold. Some events may not show up given a different background subtraction technique and a particular colour table setting. The important point is that the Harra et al. approach identifies many more blinker events than the Brković et al. approach, for the same data set.

This exercise has demonstrated that the differences in the results obtained by Harra et al. (2000) and Brković et al. (2001) are due mainly to the two different detection techniques. The determination of the duration of the events naturally changes with the different background removal and event identification schemes. This demonstrates the impact that different analysis techniques have on results, and shows that caution should be used. It does show that the results obtained from the "open slit" observation and the single slit location observation are certainly showing the same phenomena, but characterising them in different ways.

Thus, to conclude this section, we believe that it has been demonstrated that the blinkers detected using CDS rasters (Harrison, Bewsher, Parnell and co-workers), using EIT snapshot images (Berghmans and co-workers), using rapid CDS single slit observations (Harra, Gallagher and co-workers) and using CDS open slit images (Brković and co-workers) are all different observational aspects of the same event phenomenon. The different names and phrases used to describe these events, namely blinkers, quiet-Sun cell brightenings, EUV brightenings, and network brightenings should be reduced to one term. We suggest that the term blinker is now used generally to describe these events because it implies nothing about location (e.g. network, cell etc.) or the relation of the event type to any other solar phenomena (e.g. flare).

\section{Explosive events and their relationship to blinkers}

Another major small-scale, transient event-type is the explosive event, which is defined observationally by velocity shifts rather than by brightenings, in transition region ultraviolet lines. We now explore the relationship between this event-type and blinkers.

These events were detected first using the High Resolution Telescope and Spectrograph (HRTS) rocket experiment (Brueckner \& Bartoe 1983) but the identification and analysis of these events has advanced significantly using the Solar Ultraviolet Measurement of Emitted Radiation (SUMER; Wilhelm et al. 1995) experiment on board SOHO. In particular, this work has been addressed by Innes and co-workers (Innes et al. 1997a,b; Innes 2001).

These events do not have a strict definition. As noted by Innes (2001), they are small-scale (about 1500-4000 km) dynamic events that have high velocity (about $100-150 \mathrm{~km} \mathrm{~s}^{-1}$ ) wings in their line profiles. They can be found in the network and cell regions, which is a common property of blinkers. According to Innes (2001), 50\% of SUMER explosive events appear to be associated in space and time with EUV brightenings observed by CDS. However, the SUMER observations are limited to single, narrow slit locations and Innes notes that for many of the remaining events there is an EUV brightening nearby, but out of the SUMER slit field of view. 
Table 4. The properties of UV explosive events due to Innes et al. (1997).

\begin{tabular}{ll}
\hline \hline Property & Value \\
\hline Global frequency & $500 \mathrm{~s}^{-1}$ \\
Velocity & $150 \mathrm{~km} \mathrm{~s}^{-1}$ \\
Area & $2.3 \times 10^{6} \mathrm{~km}^{2}$ \\
Mean Lifetime & $60 \mathrm{~s}$ \\
\hline
\end{tabular}

If explosive events are one component of a transient event type, which includes the blinker, then our ability to link these events effectively is severely limited by the observational limitations of our instrumentation. Innes et al. (1997) give typical explosive event characteristics as in Table 4.

Let us suppose, briefly, that there is an explosive event embedded within what we think of as a blinker. The CDS observations used to detect blinkers have been made using a 4 arcsec wide slit, with 1.68 arcsec sized $(\mathrm{N}-\mathrm{S})$ pixels. The typical explosive event covers an area $2 \operatorname{arcsec} \times 2 \operatorname{arcsec}$, i.e. just $60 \%$ of a CDS pixel area. Only a fraction of that plasma is in motion, probably less than $10 \%$. Thus, some $6 \%$ of the CDS intensity for that pixel will show motion. In reality, the fraction of emitting plasma in motion may be much smaller and, we note that the SUMER spectral resolution and line widths are superior to those of CDS (which was not designed with velocity and line profile observations in mind!). Thus, we may expect that the CDS data would not readily display these types of velocity events, even if they are related to the blinkers.

To explore the relationship further, we first look to the work of Chae et al. (2000) and Innes (2001). In the former study, attempts were made to compare SUMER explosive events and CDS blinker events, and, in the latter, SUMER observations were compared to EUV images from the Transition Region and Coronal Explorer (TRACE; Handy et al. 1999). Chae et al. (2000) showed that explosive events tend to occur on the edges of, and simultaneous with, strong brightenings in O V rasters from CDS, and Innes (2001) confirmed that the C IV transition region images from TRACE, showed brightenings in association with velocity events. However, neither Chae et al. (2000) nor Innes (2001) actual determine whether the EUV brightenings they see had the same characteristics as blinkers. In particular, the "C IV images" from TRACE are actually images made up from various broadband filter images and so it is difficult to say exactly what can be seen and at what temperature the features they see are. Nonetheless, these results confirm the suggestion that explosive events generally occur in network regions, like many blinkers. Furthermore, it suggests a possible relationship between blinkers and the UV explosive events.

Bewsher (2002) and Madjarska \& Doyle (2003), however, disagree. They too look at the relation between blinkers and explosive events and come to different conclusions. Bewsher (2002) uses the same data set as Innes (2001) and in fact compares the explosive events Innes (2001) finds with blinkers she has detected in the simultaneous $\mathrm{O} V$ data from CDS. Clearly, since the SUMER data are only single slit and the CDS data are for rastered images, the cadences of the data sets are very different and their spatial overlap small. Nevertheless, Bewsher (2002) manages to identify one event where both a blinker and explosive event occurs. The explosive event was on the edge of the blinker; it, of course, lasted for only a fraction of the time that the blinker did and the blinker did not show enhanced wings like the explosive event, as predicted above. However, Bewsher (2002) also find 7 other explosive events events all of which were not connected with a blinker. Furthermore, she also finds blinkers with no associated explosive event. In all cases the events occurred in the network possibly explaining why other authors believe their explosive events are connected with blinkers.

Madjarska \& Doyle (2003), use a different approach to analyse the relationship between blinkers and explosive events. A specially designed joint observing sequence involving CDS O V and SUMER Ne V data was used. From the O V rastered images they first identified events by eye that had the same typical characteristics as blinkers. Then these data were compared with simultaneous $\mathrm{Ne} \mathrm{V}$ single slit data which is at a temperature of $2 \times 10^{5} \mathrm{~K}$, just below the temperature of the $\mathrm{O} V$ line. From this analysis they identified 3 blinkers, first using the $\mathrm{O} \mathrm{V}$ rasters and then in the $\mathrm{Ne} \mathrm{V}$ data, and 2 explosive events in $\mathrm{Ne} \mathrm{V}$. The explosive events occurred on the edges of the $\mathrm{O} \mathrm{V}$ blinkers. However, since the Ne $\mathrm{V}$ blinkers only reflect the core brightest pixels of the $\mathrm{O} \mathrm{V}$ blinkers, and so cover a smaller area, the explosive events are not on the edges of the Ne V blinkers. Furthermore, the occurrence of the explosive events did not seem to correspond to any particular behaviour or characteristic of the blinkers, in particular, they compared the line profiles of the events. Also one blinker had no explosive event associated with it. Madjarska \& Doyle (2003), therefore, concluded that blinkers and explosive events are not related directly.

These conflicting results are still open for discussion although it does seem likely that blinkers and explosive events are not intimately related. If they are related, however, then any theory needs to explain (i) why are there so many more explosive events than blinkers, (ii) why the lifetime of explosive events are so much shorter than that of blinkers and (iii) why the $\mathrm{Ne} \mathrm{V}$ blinkers do not show velocities like those in explosive events.

The lack of any link between blinkers and explosive events should not necessarily be a surprise. Most blinkers appear to be density/filling factor events, not temperature events and are therefore very unlikely to be a direct result of reconnection. Explosive events on the other hand are associated with high velocities and they could well be directly related to reconnection as has been suggested by Innes et al. (1997a).

\section{Transient events at higher temperatures}

The discussion thus far has been limited mainly to transition region temperatures. However, Harrison et al. (1999) did report that coronal lines displayed either no effect or a small brightening during a blinker. They identified each blinker as an enhancement in the $\mathrm{O} \mathrm{V}(250000 \mathrm{~K})$ transition-region line and then looked to see the effects in other emission lines. 
The average enhancements for the million $\mathrm{K} \mathrm{Mg} \mathrm{IX} \mathrm{and} \mathrm{Mg}$ X emission lines at $368 \AA$ and $624 \AA$ were only $4 \%$ and $7 \%$, respectively, for blinkers, but some events did show increases of up to $40 \%$.

Bewsher et al. (2002) used the same method, i.e. identifying events using the $\mathrm{O} V$ data and only then examining the coincident response in the hotter Mg IX and X emission lines. Indeed, their event selection criteria were such that they did not identify any "Mg IX/X blinkers" in their data, however, 7\% and $13 \%$ of $\mathrm{O}$ V blinkers, respectively, produced signatures in $\mathrm{Mg}$ IX and Mg X that could be classed as weak events with peaks just $20 \%$ of those of a verifiable blinker.

Harra, Gallagher and co-workers (Harra et al. 2000, and Gallagher et al. 1999) did not examine any lines hotter than the O V $250000 \mathrm{~K}$ line.

In hindsight, what should have been done in these studies would have been to have searched the data independently for brightenings in each line, rather than define the event using the $\mathrm{O} \mathrm{V}$ data only. This line was used because it clearly showed the greatest enhancements, but a search through the $\mathrm{Mg}$ data, for example, for brightenings, without reference to the cooler O V data, would have been beneficial. In other words, we are examining the basic question, are there blinkers, which achieve higher temperatures?

Brković et al. (2001) did indeed search for brightenings in their data-sets for each line independently. They found a total 450, 300 and 1971 brightenings in emission from He I $584 \AA$ $(20000 \mathrm{~K}), \mathrm{O}$ V $629 \AA(250000 \mathrm{~K})$ and $\mathrm{Mg} \mathrm{IX} 368 \AA$ $(1000000 \mathrm{~K})$ respectively, from their data-sets. The Mg IX durations were in the range $2-40 \mathrm{~min}$, peaking at $5 \mathrm{~min}$ and with a mean of $12.23 \mathrm{~min}$. Only $20 \%$ of the O V blinkers coincided with Mg IX blinkers (i.e. 60 events).

This suggests that we are witnessing either (i) a single event type which commonly does not achieve coronal temperatures, but, some events can extend from transition region to coronal temperatures and some are seen only at coronal temperatures, or (ii) there are two types of blinkers and maybe even two types of higher temperature events - one set that are merely produced at transition temperatures (or for the higher temperature events merely produced in the corona) and another set that are heating/cooling events that can be viewed at different temperatures.

For the former, whether or not an individual event achieves coronal temperatures will depend on the mechanism that produces blinkers. Remember that the basic O V blinker is described as a density or filling factor enhancement, not a heating event (Harrison et al. 1999). So any mechanism would somehow have to not heat the plasma yet exist at many temperatures, transition region and coronal.

Of course, for the latter case, the hotter events would in general not be related to the EUV blinkers. However, given the fact that $20 \%$ of the Mg IX events found by Brković et al. (2001) showed a correlation with $\mathrm{O} V$ events, and that the initial studies of Harrison (1997) and Harrison et al. (1999) and Bewsher et al. (2002) did show some Mg IX signatures, it is difficult to avoid the conclusion that some EUV blinkers can generate coronal temperature enhancements, and some not.

Brković et al. (2001) identified many more Mg IX events than $\mathrm{O}$ V events. The Mg IX events showed a global birth rate of $55.4 \mathrm{~s}^{-1}$. Of course, much must depend on the selection threshold used. Also the events seen by Bewsher et al. (2002) that registered in Mg IX and Mg X were all short-lived singlepeaked events and so for these events it is impossible to say whether they follow the same pattern as the generic blinkers, i.e are density/filling factor events, and not temperature events. Thus, there is a possibility that there are two types of blinkers. Those that are related to coronal events and those that are not.

A number of authors have studied the corona, in particular quiet-coronal regions, looking for small-scale events, e.g. Krucker \& Benz (1998), Parnell \& Jupp (2000) and Aschwanden et al. (2000a,b). All these authors found smallscale transient events using various different detection techniques and data sets taken from different telescopes. Despite these differences and the fact that the authors choose to call their events by different names it is generally accepted that the network flares and heating events found using EIT and discussed by Krucker et al. (1997; see also Benz \& Krucker 1999; and Krucker \& Benz 1998) are essentially the same as the nanoflares mentioned by Parnell \& Jupp (2000) and Aschwanden et al. (2000a,b) and detected using TRACE data. The events found in EIT were not called nanoflares because they did not have energies of $10^{-9}$ less than a large solar flare, i.e., $10^{23}$ ergs. Instead they were more "microflares" and had energies between $10^{25}-10^{26}$ ergs. The events found by Parnell \& Jupp (2000) had energies as low as $10^{23}$ ergs, though they did range up to $10^{26}$ ergs. Aschwanden's events ranged between $10^{24}$ and $10^{26}$ and so almost reached nanoflare energies. Nonetheless, the practically identical techniques used by Krucker \& Benz (1998) and Parnell \& Jupp (2000) suggests that they must be the same event-type.

Krucker et al. (1997; see also Benz \& Krucker 1999; Krucker \& Benz 1998) announced the identification of an event-type in X-rays which they initially called network flares; these were later classified by the authors as "heating events". These are events which typically achieve temperatures of 1.8 million $\mathrm{K}$ in initial studies using the Yohkoh Soft X-ray Telescope (SXT; Tsuneta et al. 1991) and a global birth-rate of $0.33 \mathrm{~s}^{-1}$ was calculated. The X-ray birth-rate is much smaller than the blinker birth-rates given above, though we must consider instrument relative sensitivities in any rigorous study.

Again, much depends on the different methods for event identification. However, for the CDS observing sequences of Bewsher et al. (2002), for example, which was 12 hours in duration, for a $40 \times 124 \operatorname{arcsec}^{2}$ region of the Sun, one might have expected only 6 events to have been found in either Mg IX or $\mathrm{Mg} \mathrm{X}$ to be associated with the 356 events detected in $\mathrm{O} \mathrm{V}$, if the X-ray birth-rate is used as a guide for the number of anticipated events which we could have detected using the Mg IX or $\mathrm{Mg} \mathrm{X}$ million $\mathrm{K}$ emission lines. In reality, Bewsher et al. (2002) found 27 weak Mg IX events associated with blinkers and 46 weak Mg X events. This suggests that the lack of a signature above most of the blinkers may be real.

The work on coronal events was extended, however, to include observations from the highly ionised iron lines detected by the EIT instrument on SOHO (e.g. Benz \& Krucker 1999; and Krucker \& Benz 1998). Here, Krucker \& Benz (1998) found a considerably larger birth rate of $293 \mathrm{~s}^{-1}$, using the 
Fe IX/X and Fe XII EIT observations which are from plasmas nearer to 1 million $\mathrm{K}$. This is some 5-6 times the Mg IX birth rate found by Brković et al. (2001). This disparity may be explained by the different detection techniques, in particular, whether a sequences of peaks counts as a number of small events or one long event. Furthermore, instrument sensitivities, resolutions and cadences of the data sets will have a significant effect on the numbers of events detected.

We note that the heating event/network flares have measured areas of order $2.7 \times 10^{7} \mathrm{~km}^{2}$, which is identical to the blinker size, and that the mean network flare duration of $10 \mathrm{~min}$ is of the same order as a typical blinker duration.

The Bewsher et al. (2002) study selected events using the $\mathrm{O} \mathrm{V}$ identification of brightenings (and not the $\mathrm{Mg}$ IX intensity, independently), and was also limited to detecting events with durations of more than $300 \mathrm{~s}$ and so the limited number of events they detected may not be representative of the true numbers of coronal events associated with blinkers. Though given the fact that the Krucker and Benz EIT Fe IX/X and XII birth rates are so high, it is a little surprising that Bewsher et al. (2002) did not identify more events in the Mg IX and $\mathrm{Mg} \mathrm{X}$ lines - especially as the temperature of these ions is similar to that of Fe IX and X. Another, factor limiting the number of events detected by Bewsher et al. (2002) could be that they considered only the sum of all the coronal pixels that related to the blinker and if, for instance, the coronal event was smaller or offset from the $\mathrm{O} \mathrm{V}$ blinker its signature may be lost due to the summing of many "background" pixels. It is worth remembering that the analysis due to Brković et al. (2001) revealed a birth rate of $\mathrm{Mg}$ IX brightenings which were identified independently of the O V events and only $20 \%$ of these coincided with $\mathrm{O} V$ events. Thus, if we accept that coronal events are typically weaker than $\mathrm{O} \mathrm{V}$ blinkers, then Bewsher et al. (2002) may only have detected $1 / 3-1 / 2$ of the blinker related events.

Krucker \& Benz (2000) have further extended their work to include observations from the EIT $1600000 \mathrm{~K}$ Fe XII $195 \AA$ line and the CDS 250000 K O V $629 \AA$ line, together. They examined individual events and showed that transition region brightenings occurred minutes before associated cooler He I enhancements, which are then followed by the hotter Fe XII brightenings. They also include radio VLA observations, which show enhancements in line with the He I data. The events they studied were relatively large events, in the quiet Sun, and they suggest that the sequence of events has a lot in common with flare events, i.e. the quiet-Sun phenomenon is a small version of a regular flare. This result, however, begs the question why are blinker oxygen ratios flat? If blinkers are "flare" like events surely they would show a heating or cooling signature?

Coronal brightening signatures similar to the network flares are reported using the coronal Fe XII observations from EIT by Berghmans et al. (1998) for which they found a global birth-rate of about $1.2 \mathrm{~s}^{-1}$. A typical size of these events amounts to tens of $\mathrm{Mm}^{2}$, their duration is several minutes and radiative losses are in the range $10^{24}-10^{26}$ erg. Comparing these parameters and those of heating events/network flares
Berghmans et al. (1998) suggested that the Fe XII brightenings represent the low-energy counterpart of the network flares.

Even lower energy counterparts to network flares have been found using TRACE, and these have been called nanoflares (Parnell \& Jupp 2000). The nanoflare idea was introduced by Parker $(1981,1988)$ as a theoretical concept for localised impulsive energy releases of the order $10^{23}-10^{25} \mathrm{erg}$, i.e. approximately $10^{-9}$ that of the flare. Parker proposed that these small magnetic reconnection events may be responsible for heating the solar corona. The contribution of nanoflares to coronal heating can be estimated through the study of frequency distributions of observed nanoflare thermal energies. The frequency distributions follow a power law of the form $N(E)=N_{0} E^{-\gamma}$, where $N_{0}$ is the normalization factor. Hudson (1991) showd that if $\gamma>2$ then nanoflares can significantly contribute to the coronal heating under the strong assumption that the power law can be extrapolated to smaller energies. In the literature we do not find consistent values for the power law index $\gamma$. Studies of brightenings in a range of wavelengths have been linked to the nanoflare idea and, depending on the study, we find a large range of values for the power-law index. These include: Fe XII brightenings (Berghmans et al. 1998), $\gamma=1.35$, nanoflares (Parnell \& Jupp 2000), $\gamma=2.1-2.5$, soft solar X-ray bursts (Drake 1971), $\gamma=1.84$ and (Hudson 1991), $\gamma=1.8$ and for network flares/heating events (Krucker \& Benz 1998), $\gamma=2.3-2.6$. The non-uniqueness of these values is basically due to the fact that the analysed data were obtained with different instruments and the events are identified using different techniques.

Parnell \& Jupp (2000) used TRACE data from observations taken in the Fe IX/X $171 \AA$ and Fe XII $195 \AA$ emission lines. These are sensitive to temperatures of order $1-$ 1.5 million K. They identified between 1861 events (with areas greater than $2.11 \times 10^{6} \mathrm{~km}^{2}$ and enhancement greater than $2 \sigma$ ) and 13088 events (with area greater than $0.25 \times 10^{5} \mathrm{~km}^{2}$ and enhancements greater than $2 \sigma$ ) in each line over an observing period of about $15 \mathrm{~min}$. The area studied was just $1.2 \times 10^{11} \mathrm{~km}^{2}$ implying a global birth rate ranging from 92-727 s $\mathrm{s}^{-1}$, which bound the global birth rates identified by Brković et al. (2001) and Krucker \& Benz (1998). They are again much higher than the blinker birth rates agreeing with the suggestion that only a fraction of the coronal brightenings are associated with blinkers.

Once again these results depend greatly on the sensitivity of the instrument and the detection algorithm used. For example, Aschwanden \& Parnell (2002) used TRACE data from observations taken in the Fe IX/X $171 \AA$ and Fe XII $195 \AA$ emission lines. These are sensitive to temperatures of order $1-$ 1.5 million K. Here, however, selection effects were imposed and only 1100 events were identified in each line over an observing period of about $40 \mathrm{~min}$. Given the TRACE field of view, this projects to a global birth rate of about $23 \mathrm{~s}^{-1}$, which is very similar to the lower temperature blinker birth rate. Furthermore, due to the selection only "flare-like" events were chosen - i.e., only events that were first identified in Fe XII and then in Fe IX/X - so all of these events are supposedly cooling events and, therefore, it is not unreasonable to assume they are detectable in transition-region lines. However, the temperatures of 
these events would suggest that they should also have been detected during the blinker studies of CDS in the Mg IX emission line. Clearly, they are not, with the TRACE data giving a much higher birth rate than one might expect from the CDS data. However, the TRACE cadence of $80 \mathrm{~s}$ is a factor of 2-4 faster than CDS, allowing shorter duration events to be identified, and there may also be a significant improvement in sensitivity.

Thus, our conclusions when considering the higher temperature transient event observations are not as clear cut as for the transition region temperature events. It is possible that the higher temperature events are simply blinkers that have achieved higher temperatures - i.e. blinkers are the transitionregion signature of a heating or cooling flare-like event. This would suggest that they are related to magnetic reconnection, which is consistent with the picture given by Benz \& Krucker (2000) and from the number of events observed by Aschwanden \& Parnell (2002). However, the wildly different birth-rates of the X-ray and EIT iron ion (Krucker \& Benz 1998) and TRACE iron (Parnell \& Jupp 2000) ion events, combined with the lack of Mg IX events in some analyses of the CDS data suggest an alternative scenario in which there are (i) blinkers that are purely density/filling factor events which only occur at chromospheric/transition region temperatures and are possibly caused by compression, (ii) "flare-like" heating/cooling events that occur in a range of temperatures and (iii) coronal heating-events that do not show "flare-like" behaviour possibly because they are created by a wave heating mechanism such as resonant absorption or mode coupling. Clearly, however, much further work needs to be done before we can draw firm conclusions.

\section{Summary and discussion}

An examination of observational results and instrumental characteristics has demonstrated that a range of transient events detected at transition-region temperatures using different EUV spectral rastering and imaging techniques, and EUV narrow band imaging, are, in fact, identical. That is, events known as blinkers, network and cell brightenings, and EUV brightenings, using the SOHO CDS and EIT instruments are indeed the same phenomenon detected using different techniques. We suggest, therefore, that we adopt a single name for these events and suggest the term "blinker" is used for all of these events, to avoid any implication about location or an association with other physical processes - i.e. we avoid the use of words such as "network", "cell", "flare", "heating" etc.

Perhaps surprisingly, a review of UV explosive event observation characteristics, as well as coincident EUV observations, suggests that explosive events and blinkers are not directly related. Although, a few blinkers and explosive events have been observed to overlap spatially, with the explosive events found on the edges of the blinkers, detailed analysis indicates that there is no direct relationship. This implies that there are at least two different types of transition region phenomena. The general explanation for explosive events is magnetic reconnection causing 100-200 $\mathrm{km} \mathrm{s}^{-1}$ outflow jets or shocks which are observed as wing enhancements in the spectral line profiles. Blinkers appear to be enhancements due to density or filling factor enhancements and, even when observed using SUMER, do not show high velocity wind enhancements. This may indicate that blinkers are not created by reconnection, but by the merging of magnetic fields, which is constistent with the density/filling factor interpretation given above.

Up to $20 \%$ of blinkers show temperatures up to the $1000000 \mathrm{~K}$ range, although most events found at transitionregion temperatures do not show these high temperature values. A consideration of transient event observations above 1 million $\mathrm{K}$, including the network flare/heating event and nanoflare observations in EUV and X-ray wavelengths shows that at least some higher-temperature events are associated with blinkers. However, the majority of higher-temperature events have no transition-region signatures.

From these results there is no conclusive evidence indicating whether or not blinkers and higher-temperature events are the same phenomenon. Instead, there are two possibilities: (i) they are indeed the same event-type, driving responses in varying temperatures ranges; or (ii) there are two types of blinkers/higher-temperature events - one set that are powered by the same mechanism and have flare-like characteristics being powered by magnetic reconnection and a second set of blinker events that are density/filling-factor events which are created by, most probably, a compression mechanism. The key difference between the flare-like event-type and explosive events is likely to be where reconnection takes place. Reconnection in the transition region may produce explosive events whilst reconnection in the corona could give rise to very different behaviour and cause a flare-like event which on cooling may give rise to enhanced emission in the transition region.

A true confirmation of the conclusions of this work could be achieved through careful multi-wavelength observations using existing and future instrumentation aboard SOHO, TRACE and Solar-B, with as broad a range of temperatures as possible.

We must improve on existing statistical analyses of transient events, to establish firmly the links/differences between coronal and transition region temperatures, and between the blinkers and explosive events. This will require far longer duration sequences than have previously been run using a combination of EUV and UV instruments. In addition, the observations ought to include a co-ordinated effort involving not just the EUV/UV instruments, but a combination of imaging and spectroscopy, with as high a cadence as possible, with coincident magnetic data. Despite the need to provide high time resolution, the spectroscopic instruments must include the detection of events from as broad a temperature range as possible. All of this represents a significant upgrade of the observations taken so far using individual instruments and combinations of instruments.

Acknowledgements. SOHO is a mission of international co-operation between ESA and NASA. The need for this review was identified at a workshop dedicated to small-scale transient activity held at the Institut d'Astrophysique Spatiale, Orsay, in December 2001, chaired by Clare Parnell, with local organisation led by Karine Bocchialini. The discussions at that workshop provided valuable input to this paper. The work of $\mathrm{AB}$ was supported by the Deutsche Forschungsgemeinschaft grant No. PE 782, which is gratefully acknowledged. 


\section{References}

Aschwanden, M. J., \& Parnell, C. E. 2002, ApJ, 572, 1048

Aschwanden, M. J., Nightingale, R., Tarbell, T., \& Wolfson, C. J. 2000a, ApJ, 535, 1027

Aschwanden, M. J., Tarbell, T., Nightingale, R., et al. 2000b, ApJ, 535,1047

Berghmans, D., Clette, F., \& Moses, D. 1998, A\&A, 336, 1039

Benz, A., \& Krucker, S. 1999, A\&A, 341, 286

Bewsher, D. 2002, Ph.D. Thesis

Bewsher, D., Parnell, C., \& Harrison, R. A. 2002, Sol. Phys., 206, 21

Brković, A., Solanki, S. K., \& Rüedi, I. 2001, A\&A, 373, 1056

Brueckner, G. E., \& Bartoe, J.-D. F. 1983, ApJ, 272, 329

Chae, J., Wang, H., Goode, P. R., Fludra, A., \& Schüehle, U. 2000, ApJ, 528, L119

Delaboudiniere, J.-P., Artzner, G. E., Brunaud, J., et al. 1995, Sol. Phys., 162, 291

Drake, J. F. 1971, Sol. Phys., 16, 152

Gallagher, P. T., Phillips, K. J. H., Harra-Murnion, L. K., Baudin, F., \& Keenan, F. P. 1999, A\&A, 348, 251

Handy, B. N., Acton, L. W., Kankelborg, C .C., et al. 1999, Sol. Phys., 187,229

Harra, L. K., Gallagher, P. T., \& Phillips, K. J. H. 2000, A\&A, 362, 371
Harrison, R. A. 1997, Sol. Phys., 175, 467

Harrison, R. A., Sawyer, E. C., Carter, M. K., et al. 1995, Sol. Phys., 162,233

Harrison, R. A., Lang, J., Brooks, D. H., \& Innes, D. E. 1999, A\&A, 351,1115

Hudson, H. D. 1991, Sol. Phys., 133, 357

Innes, D. E. 2001, A\&A, 378, 1067

Innes, D. E., Inhester, B., Axford, W. I., \& Wilhelm, K. 1997a, Nature, 386,811

Innes, D. E., Brekke, P., Germerott, D., \& Wilhelm, K. 1997b, Sol. Phys., 175, 341

Krucker, S., \& Benz, A. 1998, ApJ, 501, L213

Krucker, S., \& Benz, A. 2000, Sol. Phys., 191, 341

Krucker, S., Benz, A., Acton, L. W., \& Bastian, T. S. 1997, ApJ, 488, 499

Madjarska, M. S., \& Doyle, J. G. 2003, A\&AS, 403, 731

Parker, E. N. 1981, ApJ, 244, 644

Parker, E. N. 1988, ApJ, 330, 474

Parnell, C.E., \& Jupp, P. E. 2000, ApJ, 529, 554

Parnell, C. E., Bewsher, D., \& Harrison, R. A. 2002, Sol. Phys., 206, 249

Tsuneta, S., Acton, L., Bruner, M., et al. 1991, Sol. Phys., 136, 37

Wilhelm, K., Curdt, W., Marsch, E., et al. 1995, Sol. Phys., 162, 189 